\title{
EEG Classification Using TQWT and Classifiers
}

\author{
Sibasankar Padhy ${ }^{1}$ and S Sai Suryateja ${ }^{2}$ \\ Vellore Institute of Technology, Vellore \\ ${ }^{1}$ Assistant Professor (Sr. Grade 1), School of Electronics Engineering \\ ${ }^{2} 3^{\text {rd }}$ year B Tech, Electronics and Communication Engineering
}

\begin{abstract}
The purpose of this study is to detect the epileptic seizures, which can be indicated by the abnormal disturbances in intracranial neurons using the electroencephalogram (EEG) signals. The EEG signals are grouped into three categories viz., Normal EEG signals ( $Z$ and $O$ subsets), Seizure-free EEG signals ( $N$ and $F$ subsets), and Seizure EEG signals ( $S$ subset). Whereas, for classification in this study, EEG signals are divided into three groups namely NF-S, O-F$\mathrm{S}$, and ZO-NF-S. The signal length is fixed to be 4096 samples. The EEG signals will be decomposed by using Tunable-Q Wavelet Transform (TQWT), which produces intrinsic mode functions (IMFs) in decreasing order of frequency. These IMFs are analysed to gather the features of these signals, which help to classify them into various categories, and these features are fed as inputs to three classifiers viz., Random Forest (RF), Decision Table (DT), and Logistic Regression (LR). Logistic Regression classifier has showed higher accuracy, specificity and sensitivity for NF-S and O-F-S groups in comparison to RF and DT classifiers, whereas, Random Forest classifier expressed higher accuracy, specificity and sensitivity for ZO-NF-S groups in comparison to other classifiers. By utilising $L R$ classifier, the suitable parameters of TQWT in NF-S (seizure-free vs. Seizure) are $Q=6, r=3$, and $J=9$ and showed maximum accuracy of $98 \%$; and in O-F-S (Normal vs. Seizure-free vs. Seizure), $Q=1, r=3$, and $J=9$ attained maximum accuracy of $94.7 \%$. Whereas, in ZONF-S (Normal vs. Seizure-free vs. Seizure), $Q=4, r=3$, and $J=9$ expressed maximum accuracy of $99.8 \%$ utilising Random Forest classifier.
\end{abstract}

Keywords:- Electroencephalogram, epilepsy, seizure, tunable- $Q$ wavelet transform, random forest, decision table, and logistic regression.

\section{INTRODUCTION}

Epilepsy is a chronic syndrome in which the sensory system and personae of the patients are affected. Epileptic seizure is a collection of neural disorders, which is generated due to fluctuating discharge in brain waves. Approximately, 50 million people are affected by epilepsy worldwide, of which $80 \%$ of them live in low and middleincome countries [1]. Epilepsy can be detected by the use of an electroencephalogram (EEG); and neurologists examine the EEG signals to predict the condition of the patient's brain, but it is a prolonged process and this cannot be totally accurate.

Computer-based techniques are effective, while detecting epilepsy utilising EEG signals. "Principal component analysis enhanced cosine radial basis function neural network" technique is more effective than the traditional method of visual imaging for detection of robust epilepsy and seizure [2]. Another technique named "Eigen Value Decomposition" (EVD) is used for the decomposition of EEG signals for extracting features and utilising them in time-frequency depiction method as explained by Sharma and Pachori [3].

The EEG signal compression technique like "Contextbased near-lossless compression" is better for reducing the memory space and increasing the efficiency of the computer-based diagnostic system [4]. Time-domain based prediction methods such as linear prediction filter [5] and fractional linear prediction [6] are used to detect epileptic seizures using EEG signals. Srinivasan et al., explained the extraction of time and frequency domain features, which are utilised in "Artificial Neural Network" (ANN) to detect the epileptic seizure [7]. So, there is a huge scope in making an automatic epilepsy detection system.

Another technique named "Empirical Mode Decomposition" (EMD) is used for examining EEG signals, which decomposes the signals into various Intrinsic Mode Functions (IMFs) viz., amplitude and frequency modulated signals. Pachori explained the utilisation of mean frequency of the IMFs for discriminating seizure-free and ictal-EEG signals [8]. While, Acharya et al., observed that non-linear features are more suitable for detecting the contrasts in EEG signals. Discrete Wavelet Transform (DWT) based feature extraction techniques can also be utilised for classification of EEG signals [10, 11].

$\mathrm{Fu}$ et al., explained a method viz., Hilbert-Huang (HH) transform, which can be used to compute the histogram features from time-frequency images; and to classify them into different classes [12], whereas, the technique "Tunable-Q Wavelet Transform" (TQWT) is utilised to extract features and which helps in classifying the EEG signals as focal and non-focal [13].

In this study, an attempt is made to develop a system, which can automatically identify the group of EEG signals based on the features taken from the TQWT technique. The accuracies of the features are calculated and compared using different classifiers. Normally, the dataset utilised is previous studies have two categories namely "NF-S" and "ZO-NF-S"; whereas, in this study a new dataset "O-F-S" is introduced. In "NF-S", only two groups viz., Seizurefree, and Seizure are compared, while, in "ZO-NF-S", three groups viz., Normal (200), Seizure-free (200), and Seizure (100) are compared with unequal number of signals. The new dataset "O-F-S" introduced will have dataset with 
equal number of signals in all the three groups namely, Normal, Seizure-free, and Seizure.

The plan of this study is given below:

Database and Signal decomposition technique TQWT is described in section B

$>$ Classifiers are defined and explained in section $\mathrm{C}$

$>$ Results are described in section D.

$>$ Probable conclusions are given in section E Figure 1.

Whereas, the flow chart of this study is given in

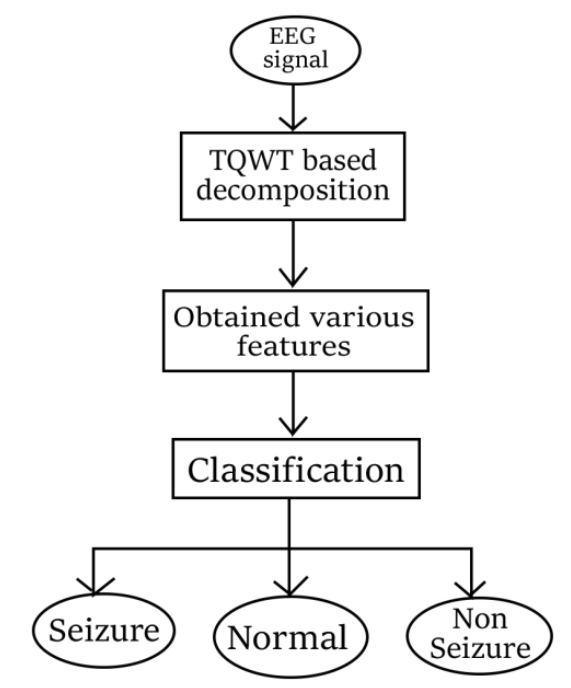

Fig 1:- Flowchart of EEG Classification

\section{DATABASE AND SYSTEM}

\section{A. Database:}

An openly available database is used in this study, as described and elaborated by Andrzejak et al., [14], which contains the recordings of healthy and epileptic patients; with 5 subsets viz., Z, O, N, F, and S. Each subset has 100 single-channelled EEG signals with duration of 23.6 seconds. The subsets $\mathrm{O}$ and $\mathrm{Z}$ are superficial EEG signals of 5 healthy subjects with eyes closed and open, respectively, which are recorded utilising a standard electrode placing scheme [14]. The subsets $\mathrm{N}$ and $\mathrm{F}$ are the EEG recordings of seizure-free signals from five recovered epileptic subjects. The $\mathrm{F}$ and $\mathrm{N}$ subsets contain the recordings of epileptogenic and non-epileptogenic zones of hippocampal formation of the brain, respectively. The $\mathrm{S}$ subset has the recordings of seizure EEG signals, which are taken from all the recording sites showing ictal properties. The 'depth electrodes' are used interracially to record the EEG signals; and the sampling rate is $173.61 \mathrm{~Hz}$ for all the EEG signals.

The EEG signals, which differentiate between normal, seizure-free and seizure subjects is clearly shown in Figure 2. The seizure signal is having rapid fluctuations, which is affecting the neural transmission and resulting in neural disorders. While, in the seizure-free signal, the disturbances are less; and the signal is slowly improving and moving towards the normal EEG signal. Wherein, the EEG signals of normal subjects will have similar differences between maxima and minima. The feature extraction and signal decomposition using TQWT is explained in next section.
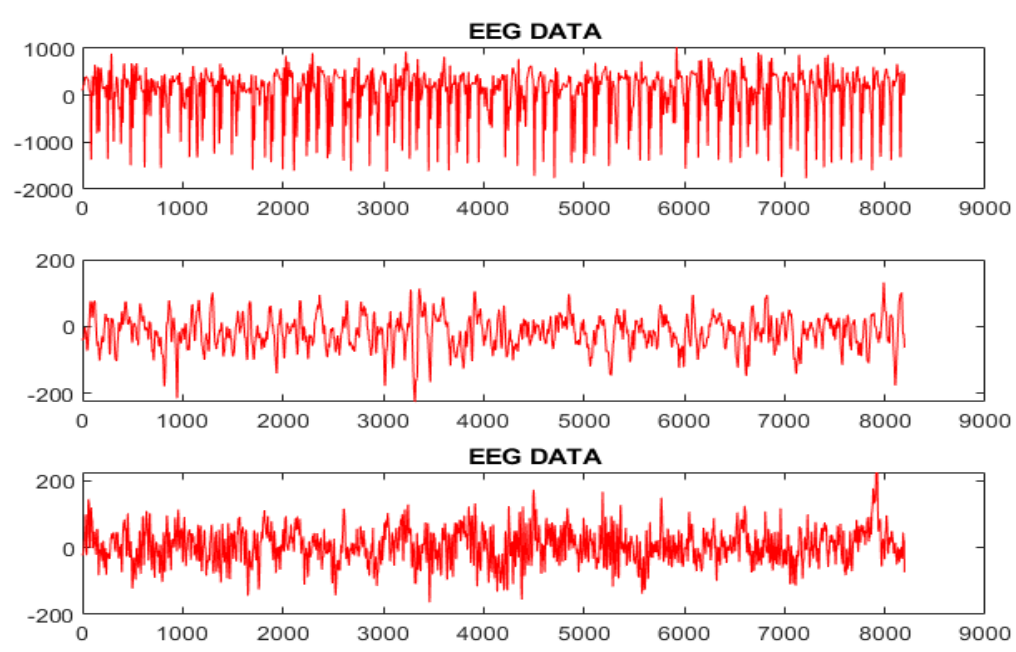

Fig 2:- EEG signals: (1) Normal, (2) Seizure-free, and (3) Seizure

\section{B. Signal Decomposition Using TQWT:}

TQWT is a widely used technique to decompose biomedical signals [16, 17], and is preferred over other methods, because it is superior to analyse the oscillatory and transient components in the signal. There are two changeable parameters utilised in this technique, $\mathrm{Q}$ and $\mathrm{r}$ namely Quality factor and redundancy, respectively [15].
The theory explained that higher the value of $\mathrm{Q}$, it is easier to extract the oscillatory nature of the signals; and lower the value of $\mathrm{Q}$; it is easier to extract the transient nature of the signals. So, it can be deduced that the resolution of time and frequency domain can be adjusted by changing the values of $\mathrm{Q}$ and $\mathrm{r}$; and the frequency domain representation of low-pass filter is used as explained by Selesnick [15]: 


$$
F_{o}(\omega)=\left\{\begin{array}{cc}
1 & , \quad|\omega| \leq(1-d) \pi \\
\theta\left(\frac{\omega+(1-d) \pi}{c+d-1}\right) & ,(1-d) \pi<|\omega|<c \pi \\
0 & , \quad c \pi \leq|\omega| \leq \pi
\end{array}\right.
$$

The representation of high-pass filter [15] in frequency domain is given as:

$$
F_{1}(\omega)=\left\{\begin{array}{cc}
1 & , \quad|\omega| \leq(1-d) \pi \\
\theta\left(\frac{c \pi-\omega}{c+d-1}\right) & ,(1-d) \pi<|\omega|<c \pi \\
0 & , \quad c \pi \leq|\omega| \leq \pi
\end{array}\right.
$$

In the above equation, $\Theta(\omega)$ is defined as follows:

$$
\theta(\omega)=\frac{[1+\cos (\omega)]}{2}[2-\cos \omega]^{\frac{1}{2}}, \quad|\omega| \leq \pi
$$

The scaling parameters of high-pass (c) and low-pass (d) filters can take values from 0 to 1 in a way that the equation, $c+d>1$ [15]. Whereas, the relation between bandwidth and Q-factor of the signal is given as follows:

$$
\mathbf{Q}=\frac{\mathbf{f}_{\mathbf{0}}}{\mathbf{b w}}
$$

In the above equation, $\mathrm{f}_{0}$ and bw symbolizes central frequency and bandwidth of IMFs, respectively. The relation between parameters $\mathrm{Q}, \mathrm{r}, \mathrm{c}$, and $\mathrm{d}$ is given below:

$$
d=\frac{2}{Q+1} \quad, \quad c=1-\frac{d}{r}
$$

The signals in Figure 2 are decomposed into IMFs using the parameter values of $\mathrm{Q}=1, \mathrm{r}=3$, and $\mathrm{J}=9$. The decomposed signals viz., IMF1 (highest frequency) to IMF 10 (lowest frequency) are depicted in Figures 3, 4, and 5; of which only IMFs 6 to 10 are used to differentiate the signals into categories because important features are present in lower frequency IMFs.

The classifiers and the statistical parameters are explained in the next section.

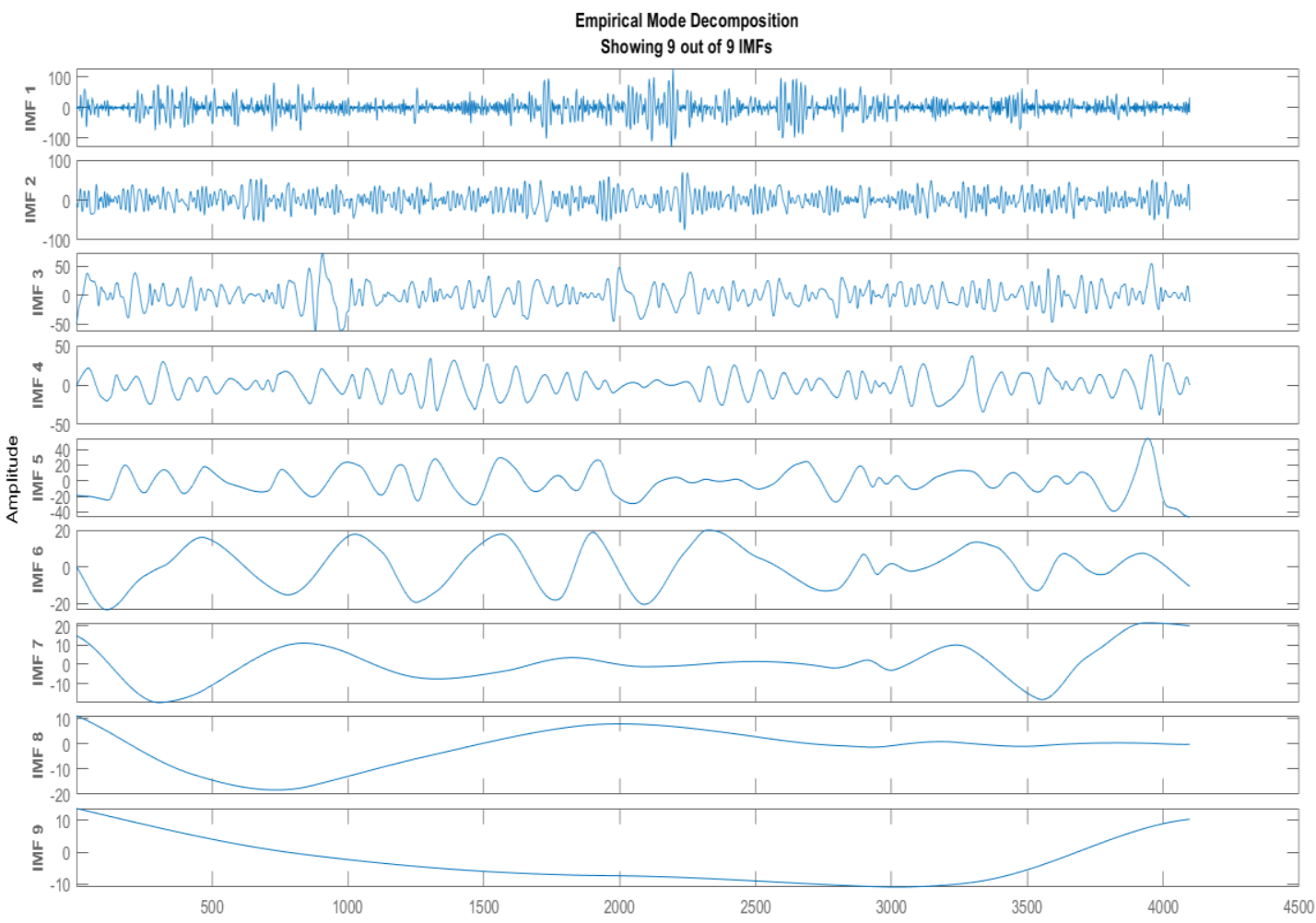

Fig 3:- IMFs of O (Normal) subset 


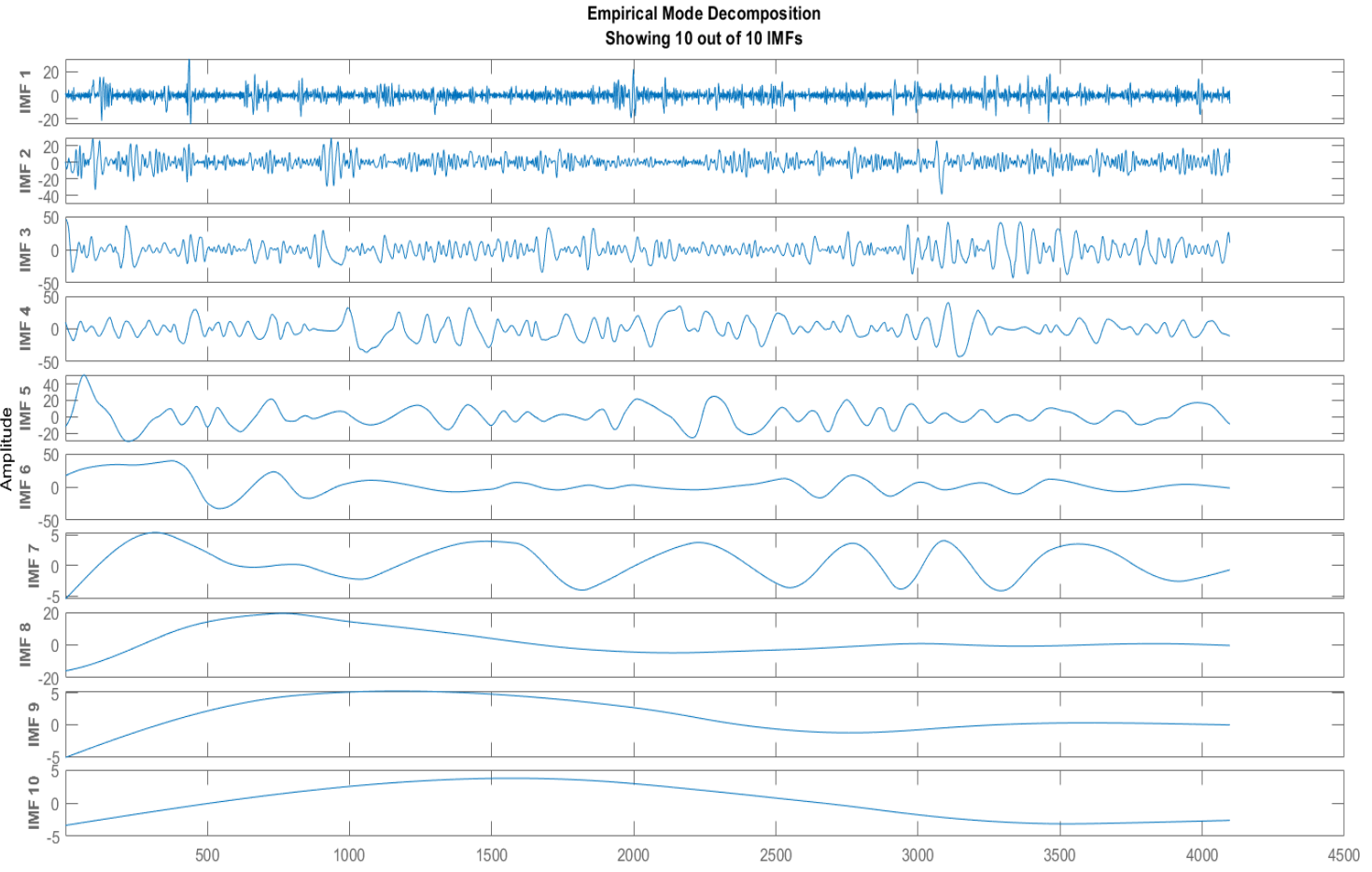

Fig 4:- IMFs of F (Seizure-free) subset

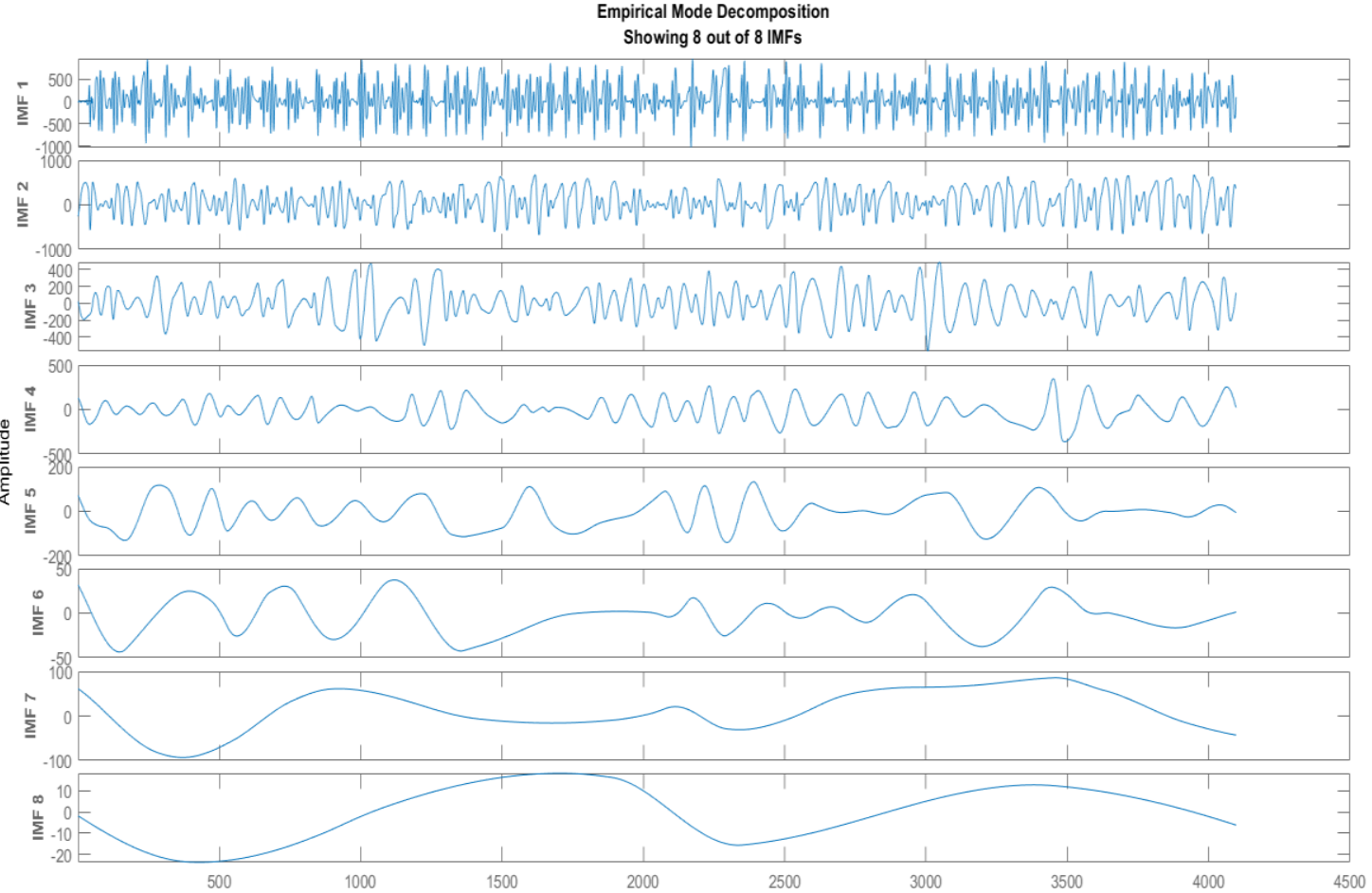

Fig 5:- IMFs of S (Seizure) subset 


\section{CLASSIFICATION}

A software named "Waikato environment knowledge analysis" (WEKA) [18] is used in this study for the classification of EEG signals utilising in-built classifiers. The classifiers used in the WEKA software are as follows:

\section{Random Forest Classifier $(R F)$ :}

This classifier is a collection of various tree classifiers. The result is decided by taking the outcome of each tree and assigning them with a weight. So, each tree is given a random vector $\delta_{\mathrm{n}}$, where $\mathrm{n}$ symbolises each tree. The random vector $\delta_{\mathrm{n}}$ is generated on its own depending on its previous vectors. So, the random forest is grown from the previous random vectors $\delta_{\mathrm{n}}$ and the training data $\mathrm{x}$. So based on the growth of the tree, the classifier $\mathrm{H}\left(\mathrm{x}, \delta_{\mathrm{n}}\right)$ is developed. So, class label is defined by using the margin function (MG).

\section{Decision Table Classifier (DT):}

This classifier is proven to be better than all decision trees for numeric predictions. The classifier follows the rule of 'If-Then', which has a great potential in both compatibility and understand-ability. It is simpler and easy to compute algorithm, which is better than decision treebased algorithm. It works on the first search and crossvalidation methods for computation. The nearest-neighbour method is used to determine the maximum of each instance in the class rather than the maximum value of the table for the same set of features.

\section{Logistic Regression Classifier (LR):}

This classifier is widely used as a statistical model for defining three parameters viz., specificity, sensitivity and accuracy, as given by Yu et al., [18]:

$$
\begin{aligned}
\text { Accuracy }(\%) & =\frac{T P+T N}{T P+T N+F P+F N} \times 100 \\
\text { Sensitivity }(\%) & =\frac{T P}{T P+F N} X 100 \\
\text { Specificity }(\%) & =\frac{T N}{T N+F P} X 100
\end{aligned}
$$

where, TP, TN, FP, and FN are symbols for true positive, true negative, false positive and false negative subjects, respectively. In this study, the logistic regression is used with the tenfold cross validation.

Comparison among the classifiers: The parameters namely specificity, sensitivity and accuracy as defined in logistic regression classifier are utilised to compare with other classifiers as mentioned above for identifying the efficient classifier.

\section{RESULTS}

In this study, the database is divided into three groups. The groups are explained as follows:

\section{Seizure-free vs. Seizure (NF-S) EEG Signals:}

The EEG signals are from three subsets viz., N (seizure-free- non-epileptogenic zone), F (seizure-freeepileptogenic zone), and S (seizure), which are sorted into two different classes that are seizure-free and seizure. So, the seizure-free class has 200 signals, while seizure class has 100 signals. At the beginning, the signals of those subsets are decomposed using TQWT. The value of parameter ' $r$ ' is fixed as 3 as suggested by Selesnick [19]. So, to find the remaining two parameters viz., Q and J (No. of stages), by fixing parameter $\mathrm{Q}$ as 1 , the value of $\mathrm{J}$ can vary from 1 to 11 . Then, the accuracy is compared for all signals for parameter value of $\mathrm{J}$ from 1 to 11 . By choosing a suitable value of $\mathrm{J}$, parameter $\mathrm{Q}$ is selected in such a way that the accuracy is the highest. So, the suitable parameters are $\mathrm{Q}=6, \mathrm{r}=3$, and $\mathrm{J}=9$ for classifying the NF-S classes of EEG signals. The classification is performed by all the classifiers listed namely, RF, DT, and LR (Table 1 and 2, Graph 1).

LR showed significant difference and higher accuracy, sensitivity and specificity in comparison to RF and DT, whereas, RF also showed significant difference and higher accuracy, sensitivity and specificity in comparison to DT. Therefore, LR is more efficient classifier compared to the other two classifiers.

Normal vs. Seizure-free vs. Seizure (O-F-S) EEG Signals:

The EEG signals taken from three subsets viz., $\mathrm{O}$ (Normal), F (Seizure-free), and S (Seizure), which are sorted into three different classes i.e., normal, seizure-free, and seizure. So, all the classes have 100 signals each. The method to select the parameters remains the same as defined in the previous section. So, the suitable values of the parameters are $\mathrm{Q}=1, \mathrm{r}=3$, and $\mathrm{J}=9$ for classifying the $\mathrm{O}$ F-S classes of EEG signals. The classification is performed by all the classifiers listed namely, RF, DT, and LR (Table 1 and 2, Graph 2).

LR showed significant difference and higher accuracy, sensitivity and specificity in comparison to RF and DT, whereas, RF also showed significant difference and higher accuracy, sensitivity and specificity in comparison to DT. Therefore, LR is more efficient classifier compared to the other two classifiers. 
Normal vs. Seizure-free vs. Seizure (ZO-NF-S) EEG Signals:

The EEG signals are from five subsets viz., $Z$ (Normal), O (Normal), N (Seizure-free), F (Seizure-free), and $\mathrm{S}$ (Seizure), which are sorted into three different classes i.e., normal, seizure-free and seizure. So, the normal class and the seizure-free class have 200 signals each; and 100 signals in seizure class. The method to select the parameters remains the same as defined in section 1. So, the suitable values of the parameters are $Q=4, r=3$, and $J=9$.
The classification is performed by all classifiers listed namely, RF, DT, and LR (Table 1 and 2, Graph 3).

RF showed significant difference and higher accuracy, sensitivity and specificity in comparison to LR and DT, whereas, LR also showed significant difference and higher accuracy, sensitivity and specificity in comparison to DT. Therefore, RF is more efficient classifier compared to the other two classifiers.

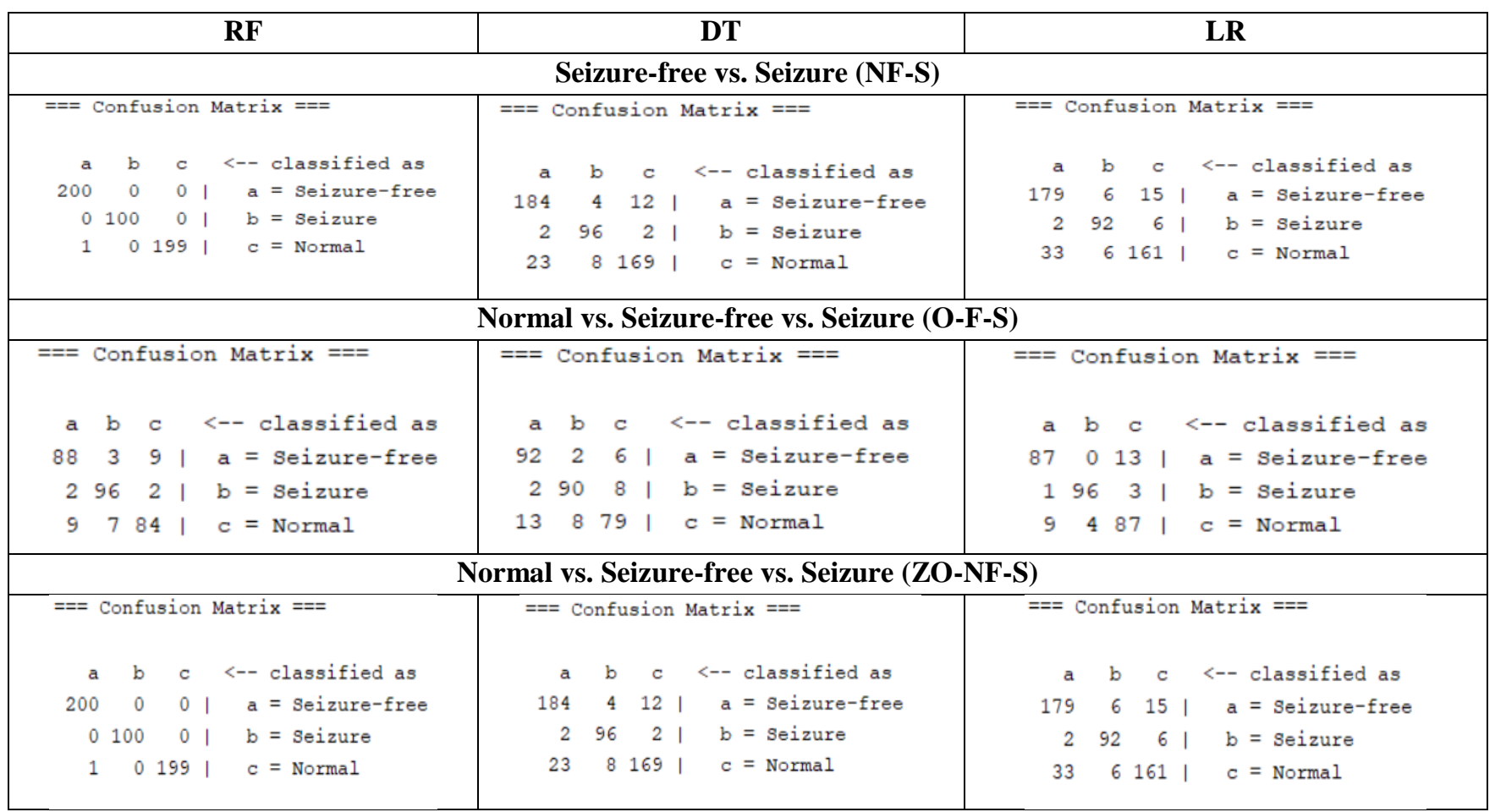

Table 1:- Confusion matrix of classifiers on different groups.

\begin{tabular}{|c|c|c|c|c|}
\hline Classes & Classifiers & $\begin{array}{c}\text { Accuracy } \\
(\%)\end{array}$ & $\begin{array}{c}\text { Sensitivity } \\
(\%)\end{array}$ & $\begin{array}{c}\text { Specificity } \\
(\%)\end{array}$ \\
\hline \multirow{3}{*}{ Seizure-free vs. Seizure (NF-S) } & $\mathrm{RF}$ & $97.0 \pm 0.07$ & $96.0 \pm 0.07$ & $95.5 \pm 0.07$ \\
\hline & DT & $93.7 \pm 0.12$ & $94.5 \pm 0.12$ & $92.0 \pm 0.12$ \\
\hline & $\overline{\mathrm{LR}}$ & $97.7 \pm 0.03$ & $98.5 \pm 0.03$ & $96.0 \pm 0.03$ \\
\hline \multirow{3}{*}{$\begin{array}{l}\text { Normal vs. Seizure-free vs. Seizure } \\
\text { (O-F-S) }\end{array}$} & $\mathrm{RF}$ & $94.3 \pm 0.09$ & $94.3 \pm 0.09$ & $94.4 \pm 0.09$ \\
\hline & DT & $89.3 \pm 0.16$ & $89.3 \pm 0.16$ & $89.6 \pm 0.16$ \\
\hline & LR & $94.7 \pm 0.05$ & $94.7 \pm 0.05$ & $94.7 \pm 0.05$ \\
\hline \multirow{3}{*}{$\begin{array}{c}\text { Normal vs. Seizure-free vs. Seizure } \\
\text { (ZO-NF-S) }\end{array}$} & $\mathrm{RF}$ & $99.8 \pm 0.02$ & $99.7 \pm 0.02$ & $99.8 \pm 0.02$ \\
\hline & DT & $90.8 \pm 0.12$ & $90.8 \pm 0.12$ & $90.8 \pm 0.12$ \\
\hline & $\begin{array}{l}\text { LR } \\
\end{array}$ & $95.4 \pm 0.05$ & $95.4 \pm 0.05$ & $95.4 \pm 0.05$ \\
\hline
\end{tabular}

Table 2:- Precision results using different classifiers 

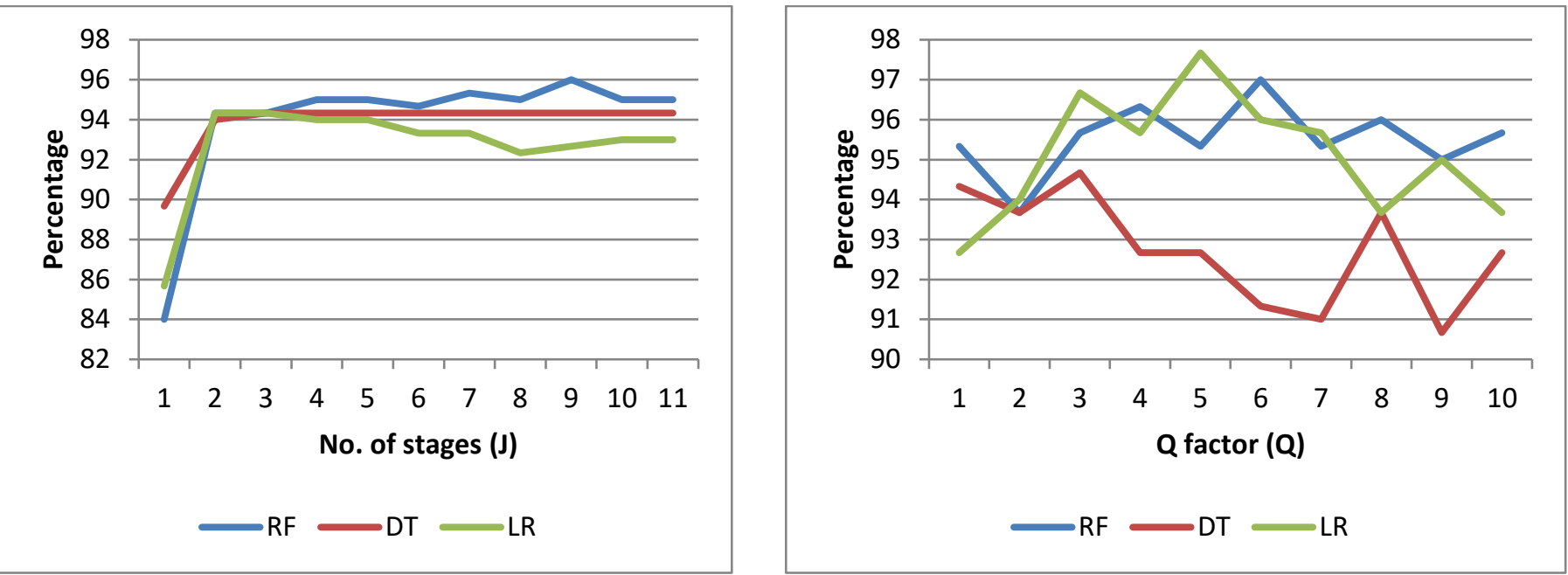

Graph 1: Seizure-free vs. Seizure (NF-S)
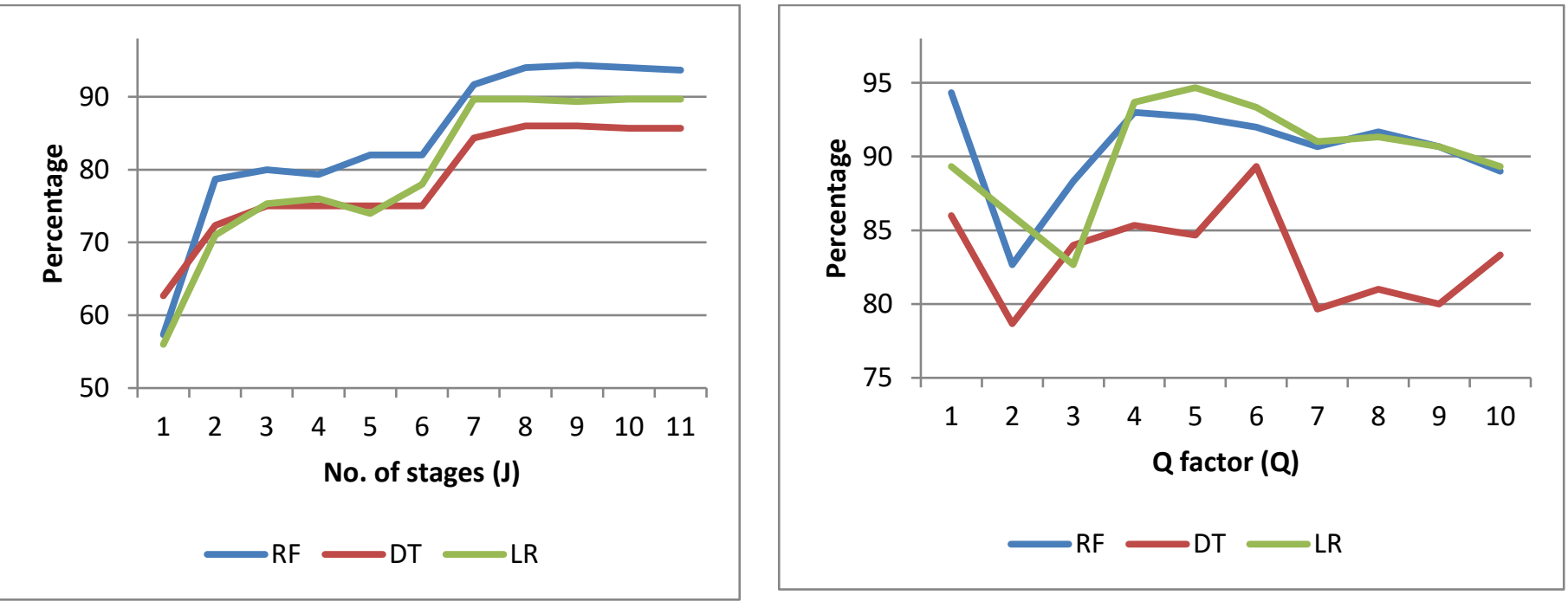

Graph 2: Normal vs. Seizure-free vs. Seizure (O-F-S)
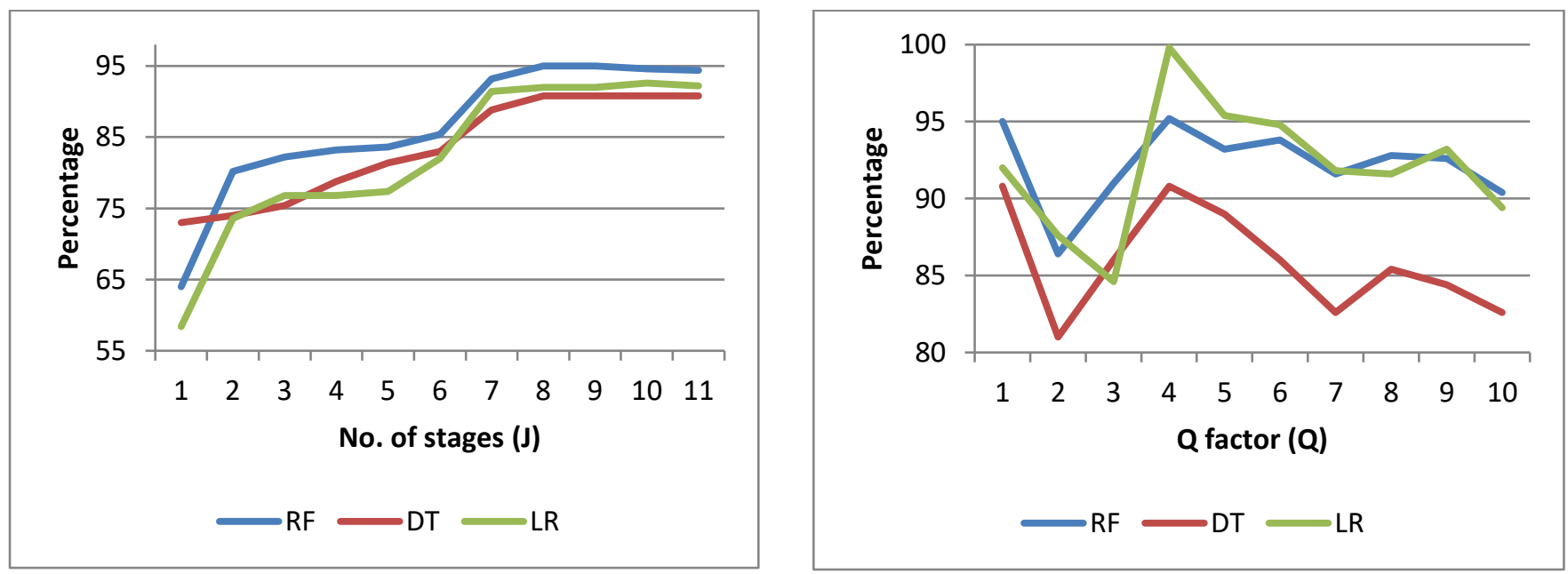

Graph 3: Normal vs. Seizure-free vs. Seizure (ZO-NF-S) 


\section{CONCLUSION}

In this study, a programmed system is used for identification of seizure using the EEG signals. The proposed system uses the TQWT algorithm to decompose the signals into features, which can be used to classify the signals. To maximise the accuracy of the system, suitable values of $\mathrm{Q}, \mathrm{r}$, and $\mathrm{J}$ are selected, which are variable for different set of signals. Three classifiers were used viz., RF, DT, and LR, for cross-validation method with ten-folds.
The accuracy of the programmed system was tested by this method. So, the highest accuracy $(98.0 \%)$ for NF-S is attained by using the parameters $\mathrm{Q}=3, \mathrm{r}=3$, and $\mathrm{J}=9$, using LR classifier. Whereas, the suitable values for O-F-S are $\mathrm{Q}=1, \mathrm{r}=3$, and $\mathrm{J}=9$, resulting in $94.7 \%$ accuracy using the LR classifier. At last, $99.8 \%$ accuracy is attained by using the parameter values, $\mathrm{Q}=2, \mathrm{r}=3$, and $\mathrm{J}=9$ in $\mathrm{ZO}-\mathrm{NF}-\mathrm{S}$ class using RF classifier. The system developed in present study is compared with earlier references (Table 3).

\begin{tabular}{|c|c|c|c|c|}
\hline Authors & Methodology & $\begin{array}{c}\text { Data set } \\
\text { combination }\end{array}$ & $\begin{array}{l}\text { Signal length } \\
\text { (samples) }\end{array}$ & $\begin{array}{c}\text { Performance } \\
\text { (accuracy) }\end{array}$ \\
\hline $\begin{array}{c}\text { Tzallas et al. } \\
(2007)[20] \\
\end{array}$ & ANN and TF features & ZO-NF-S & 4097 & $97.72 \%$ \\
\hline $\begin{array}{l}\text { Acharya et al. } \\
\text { (2012) [21] }\end{array}$ & $\begin{array}{c}\text { Entropy - Approximate, sample and phase } \\
\text { using SVM }\end{array}$ & ZO-NF-S & 4096 & $98.10 \%$ \\
\hline Peker et al. (2015) [22] & $\begin{array}{l}\text { Complex valued neural network and Dual- } \\
\text { tree complex wavelet transform }\end{array}$ & ZO-NF-S & 4096 & $98.28 \%$ \\
\hline $\begin{array}{l}\text { Bhattacharya et al. } \\
\text { (2017) [16] }\end{array}$ & k-NN entropy, TQWT and SVM & $\mathrm{ZO}-\mathrm{NF}-\mathrm{S}$ & 4000 & $98.60 \%$ \\
\hline \multirow{2}{*}{$\begin{array}{l}\text { Tiwari et al. } \\
(2016) \text { [23] }\end{array}$} & \multirow{2}{*}{ SVM and Key point local binary pattern } & NF-S & 1000 & $97.79 \%$ \\
\hline & & ZO-NF-S & 1000 & $96.71 \%$ \\
\hline \multirow{2}{*}{ Reddy et al. (2017) [24] } & \multirow{2}{*}{ TQWT, CCE RF, MLP, and LR } & NF-S & 1000 & $98.30 \%$ \\
\hline & & ZO-NF-S & 1000 & $98.20 \%$ \\
\hline \multirow{3}{*}{ PROPOSED METHOD } & \multirow{3}{*}{ TQWT, RF, DT, and LR } & NF-S & 4096 & $98.00 \%$ \\
\hline & & $\mathrm{O}-\mathrm{F}-\mathrm{S}$ & 4096 & $94.70 \%$ \\
\hline & & ZO-NF-S & 4096 & $99.80 \%$ \\
\hline
\end{tabular}

Table 3:- Comparison of present work with previous references

In future, the aim is to use this system on a larger dataset with greater number of subjects for its consistency of result; and to make the system real-time ready to reduce the time and increase the efficiency of the classifier as well as the system. This system can be applicable for various other neurological linked conditions using any biomedical signals such as EEG, ECG, and PPG etc.

\section{REFERENCES}

[1]. WHO declared fact-sheet for epilepsy, 2019, refers to the link: https://www.who.int/news-room/factsheets/detail/epilepsy

[2]. S. Ghosh-Dastidar, H. Adeli and N. Dadmehr, "Principal Component Analysis-Enhanced Cosine Radial Basis Function Neural Network for Robust Epilepsy and Seizure Detection," in IEEE Transactions on Biomedical Engineering, vol. 55, no. 2, pp. 512-518, Feb. 2008.

[3]. R. R. Sharma and R. B. Pachori, "Time-frequency representation using IEVDHM-HT with application to classification of epileptic EEG signals," in IET Science, Measurement \& Technology, vol. 12, no. 1, pp. 72-82, 12018.
[4]. N. Sriraam, Context-based near-lossless compression of EEG signals using neural network predictors,AEU International Journal of Electronics and Communications Volume 63, Issue 4, 3 April 2009, Pages 311-320

[5]. Lajnef, Tarek, Chaibi, Sahbi, Kachouri, Abdennaceur, Samet, Mounir. (2010). Epileptic seizure detection using linear prediction filter.

[6]. V. Joshi; R. B. Pachori; A. Vijesh, Classification of ictal and seizure-free EEG signals using fractional linear prediction, Biomedical Signal Processing and Control (2014), ISSN: 1746-8094, Vol: 9, Issue: 1, Page: $1-5$

[7]. V. Srinivasan, C. Eswaran, N. Sriraam. (2006). Artificial Neural Network Based Epileptic Detection Using Time-Domain and Frequency-Domain Features. Journal of medical systems. 29. 647-60. 10.1007/s10916-005-6133-1.

[8]. Pachori, Ram. (2008). Discrimination between ictal and seizure-free EEG signals using empirical mode decomposition. Research Letters in Signal Processing. 2008. 10.1155/2008/293056.

[9]. U. R. Acharya, V. S. Subbhuraam, S. Goutham, R. Martis, J. Suri. (2013). Automated EEG analysis of 
epilepsy: A review. Knowledge-Based Systems. 45. 147-165. 10.1016/j.knosys.2013.02.014.

[10]. A. Subasi. (2007). EEG signal classification using wavelet feature extraction and a mixture of expert model. Expert Systems with Applications 32, 10841093. Expert Systems with Applications. 32. 10841093. 10.1016/j.eswa.2006.02.005.

[11]. R. Upadhyay, P. Padhy, P. Kankar. (2016). A comparative study of feature ranking techniques for epileptic seizure detection using wavelet transform. Computers \& Electrical Engineering. 53. 10.1016/j.compeleceng.2016.05.016.

[12]. Fu, Kai; Qu, Jianfeng; Chai, Yi; Dong, Yong, Classification of seizure based on the time-frequency image of EEG signals using HHT and SVM, Biomedical Signal Processing and Control, ISSN: 1746-8094, Vol: 13, Issue: 1, 15-22, 2014

[13]. R. Sharma; M. Kumar; R. B. Pachori; U. R. Acharya, Decision support system for focal EEG signals using tunable- Q wavelet transform, Journal of Computational Science, ISSN: 1877-7503, Vol: 20, Page: 52-60, 2017

[14]. R. Andrzejak, K. Lehnertz, F. Mormann, C. Rieke, P. David, C. Elger. (2002). Indications of nonlinear deterministic and finite-dimensional structures in time series of brain electrical activity: Dependence on recording region and brain state. Physical review. E, Statistical, nonlinear, and soft matter physics. 64 . 061907. 10.1103/PhysRevE.64.061907.

[15]. I. W. Selesnick, "Wavelet Transform With Tunable QFactor," in IEEE Transactions on Signal Processing, vol. 59, no. 8, pp. 3560-3575, Aug. 2011.

[16]. Bhattacharyya, A.; Pachori, R.B.; Upadhyay, A.; Acharya, U.R. Tunable-Q Wavelet Transform Based Multiscale Entropy Measure for Automated Classification of Epileptic EEG Signals. Appl. Sci. 2017, 7, 385.

[17]. S. Patidar; R. B. Pachori; U. R. Acharya. Automated diagnosis of coronary artery disease using tunable-Q wavelet transform applied on heart rate signals, Knowledge-Based Systems, ISSN: 0950-7051, Vol: 82, Page: 1-10, 2015

[18]. Yu Hen Hu, S. Palreddy and W. J. Tompkins, "A patient-adaptable ECG beat classifier using a mixture of experts approach," in IEEE Transactions on Biomedical Engineering, vol. 44, no. 9, pp. 891-900, Sept. 1997.

[19]. Selesnick, Ivan. (2011). Wavelet Transform With Tunable Q-Factor. IEEE Transactions on Signal Processing. $59 . \quad 3560-3575$. 10.1109/TSP.2011.2143711. Available at: http://eeweb.poly.edu/iselesni/TQWT/

[20]. Tzallas, Alexandros \& Tsipouras, Markos \& Fotiadis, Dimitrios. (2007). Automatic Seizure Detection Based on Time-Frequency Analysis and Artificial Neural Networks. Computational intelligence and neuroscience. 2007. 80510. 10.1155/2007/80510.

[21]. Acharya, U Rajendra \& Molinari, Filippo \& Subbhuraam, Vinitha Sree \& Chattopadhyay, Subhagata \& Kh, Ng \& Suri, Jasjit. (2012). Automated diagnosis of epileptic EEG using entropies. Biomedical Signal Processing and Control. 7. 401-408. 10.1016/j.bspc.2011.07.007.

[22]. M. Peker, B. Sen and D. Delen, "A Novel Method for Automated Diagnosis of Epilepsy Using ComplexValued Classifiers," in IEEE Journal of Biomedical and Health Informatics, vol. 20, no. 1, pp. 108-118, Jan. 2016.

[23]. A. K. Tiwari, R. B. Pachori, V. Kanhangad and B. K. Panigrahi, "Automated Diagnosis of Epilepsy Using Key-Point-Based Local Binary Pattern of EEG Signals," in IEEE Journal of Biomedical and Health Informatics, vol. 21, no. 4, pp. 888-896, July 2017.

[24]. ravi shanakar, Gosula \& Rao, Rameshwar. (2017). Automated identification system for seizure EEG signals using tunable-Q wavelet transform. Engineering Science and Technology, an International Journal. 20. 10.1016/j.jestch.2017.11.003. 